\title{
Politique
}

\section{Les questions écrites dans le processus parlementaire}

\section{Maurice Champagne}

Volume 1, numéro 2, automne 1982

Les médias et les pouvoirs

URI : https://id.erudit.org/iderudit/040406ar

DOI : https://doi.org/10.7202/040406ar

Aller au sommaire du numéro

Éditeur(s)

Société québécoise de science politique

ISSN

0711-608X (numérique)

Découvrir la revue

Citer cette note

Champagne, M. (1982). Les questions écrites dans le processus parlementaire. Politique, 1(2), 143-152. https://doi.org/10.7202/040406ar d'utilisation que vous pouvez consulter en ligne.

https://apropos.erudit.org/fr/usagers/politique-dutilisation/ 


\title{
Les questions écrites dans le processus parlementaire
}

\author{
Maurice Champagne \\ Bibliothèque de l'Assemblée nationale du Québec
}

On peut distinguer un régime démocratique d'un régime totalitaire par le degré de contrôle que le peuple ou ses représentants exercent sur le gouvernement. Plus le régime permet des contrôles réels, plus il est démocratique.

$\mathrm{Si}$, en régime démocratique, on reconnaît au gouvernement le droit d'expliquer ses actes, l'opposition possède celui d'en trouver les lacunes, les faiblesses. Pour ce faire, les opposants, dans un système parlementaire de type britannique, jouissent d'une certaine latitude.

Au Québec, les députés possèdent plusieurs moyens pour contrôler les actes du gouvernement, entre autres la question avec débat, les questions orales et écrites. Ces dernières n'ayant à peu près pas été l'objet d'analyse, nous avons voulu par le présent travail corriger quelque peu cette lacune.

Un député peut poser des questions à un ministre pour obtenir des renseignements; toutefois, les questions dont les réponses exigent une certaine recherche doivent être écrites ${ }^{1}$.

Non seulement les questions écrites constituent pour les députés un moyen de se procurer des informations, mais 
elles sont également utilisées comme un moyen de contrôle des actes gouvernementaux: en fournissant une série d'informations concernant son administration ou la politique du gouvernement, le ministre a l'impression d'être contrôlé et, effectivement, il l'est.

Lorsqu'on aborde ce sujet, certaines interrogations nous viennent à l'esprit. Les questions écrites représentent-elles une procédure assez utilisée par tous les partis politiques? Quels sont les sujets touchés et les ministères les plus sollicités? Ces questions reçoivent-elles des réponses? Le délai de réponse est-il acceptable? Les questions écrites bénéficient-elles d'une certaine diffusion?

Pour répondre à ces interrogations, nous avons choisi d'analyser une session relativement longue, soit la quatrième session de la trente et unième législature, qui s'étend du 6 mars 1979 au 18 juin 1980.

La quatrième session de la trente et unième législature comptait 115 séances. Au cours de cette période les partis politiques ont posé 73 questions écrites. Le Parti libéral du Québec en a soulevé trente-cinq, l'Union nationale, vingt-neuf, le Parti québécois, six et les Démocrates, trois.

En considérant uniquement le nombre de députés des deux principaux partis de l'opposition durant cette période, soit le P.L.Q. avec vingt-six députés et l'U.N. avec dix députés, on constate que l'U.N. s'est montrée beaucoup plus intéressée que le P.L.Q. dans le domaine des questions écrites. En effet, l'U.N. avec seulement $9 \%$ de la représentation en Chambre posait $40 \%$ des questions écrites, alors que le P.L.Q. avec $24 \%$ n'en soulevait que $48 \%$.

D'autre part, le rôle traditionnel des députés ministériels (qui n'est pas de contrôler le gouvernement) explique la faible participation des députés du P.Q. D'ailleurs, des six questions 
écrites soulevées par ce parti, deux portaient sur l'administration précédente.

Les deux ministères les plus sollicités au cours de la session étudiée furent, dans l'ordre, le ministère de l'Agriculture avec dix-sept questions écrites et le ministère des Transports avec dix. M. Jean Garon était le titulaire du premier, tandis que MM. Lucien Lessard et Denis de Belleval se sont succédés aux Transports.

À l'opposé, plusieurs autres ministères furent l'objet seulement d'une à trois questions écrites de la part de l'opposition.

Il est intéressant de signaler que les ministères du Travail et de la Main-d'œuvre, de l'Immigration, de la Fonction publique n'ont eu à répondre à aucune question. De même les ministres d'État et le ministre délégué aux Affaires parlementaires n'ont pas eu à répondre.

\section{Sujets des questions écrites}

Pour connaître le sujet des questions écrites, nous les avons classées selon les quatre grandes missions de l'État: la mission économique, la mission gouvernementale et administrative, la mission éducative et culturelle et enfin la mission sociale.

Le tableau 1 indique clairement que le sujet favori des questions écrites des parlementaires touchait à la mission gouvernementale et administrative. Cette dernière fut l'objet de $67 \%$ des questions écrites, suivie de très loin par la mission économique qui a récolté $18 \%$. Le domaine de l'éducation et de la culture, de même que le domaine social n'ont suscité, ensemble, que $15 \%$ des questions.

L'intérêt à l'égard de la mission gouvernementale et administrative fut partagé non seulement entre les deux principaux partis de l'opposition, mais également par le parti ministériel. 
TABLEAU 1

Répartition des questions écrites selon les partis et le sujet des questions

\begin{tabular}{|c|c|c|c|c|c|}
\hline MISSION & P.L.Q. & U.N. & P.Q. & AUTRE & TOTAL \\
\hline Économique & $6(17 \%)$ & $4(14 \%)$ & - & $3(100 \%)$ & $13(18 \%)$ \\
\hline $\begin{array}{l}\text { Gouvernementale } \\
\text { et adm. }\end{array}$ & $23(64 \%)$ & $21(72 \%)$ & $5(100 \%)$ & - & $49(67 \%)$ \\
\hline $\begin{array}{l}\text { Éducative et } \\
\text { culturelle }\end{array}$ & $1(3 \%)$ & $3(10 \%)$ & - & - & $4(5 \%)$ \\
\hline Sociale & $6(17 \%)$ & $1(3 \%)$ & - & - & $7(10 \%)$ \\
\hline
\end{tabular}

Les questions concernant un individu en particulier ont constitué plus de la moitié des questions sur la mission gouvernementale et administrative, soit 25 sur un total de 49. Par exemple, M. Cordeau, député de l'U.N., voudrait savoir si $\mathrm{M}^{\mathrm{e}}$ «X» est à l'emploi du gouvernement? Si oui, la date de son engagement? sa fonction? son salaire? s'il a été choisi à la suite d'un concours?

Le député étant le représentant d'une circonscription électorale, on devrait s'attendre à ce que les problèmes régionaux le préoccupent. On dénombre, au cours de la période analysée, 15 questions écrites ayant un caractère régional sur un total de 73 , soit $21 \%$.

\section{Délai et taux de réponse}

En Belgique, le gouvernement dispose de 15 jours pour répondre à une question écrite. En France, en Finlande et aux Pays-Bas, le délai réglementaire est d'un mois.

Toutefois ces courts délais sont souvent dépassés. Par exemple en Belgique, 
la proportion des questions demeurées sans réponse après le délai prescrit, auxquelles il n'est répondu que tardivement, est très forte et atteint même $50 \%^{2}$.

De même en France, en 1979, on n'a répondu qu'à $6 \%$ seulement des questions dans le premier mois et $28 \%$ au cours du deuxième. La situation s'est aggravée ces dernières années :

au fur et à mesure des années, la proportion des réponses obtenues hors délai ne cesse de croître; ainsi, les réponses obtenues après plus de trois mois, qui représentaient $18 \%$ du total des réponses en 1969 , représentaient $41 \%$ en $1977 / \ldots /$ et également $41 \%$ en $1979 \ldots{ }^{3}$.

$\mathrm{Au}$ Québec, le député qui veut soulever une question écrite doit déposer au préalable un avis ${ }^{4}$. Cependant le Règlement ne précise aucun délai à l'intérieur duquel doivent répondre les ministres.

Selon le tableau 2, en 1979-1980, les ministres du Québec ont répondu à $9 \%$ des questions dans le premier mois, à $25 \%$ dans le second, à $21 \%$ dans le troisième, à $21 \%$ également au cours du quatrième et cinquième mois, et enfin à $24 \%$ des questions après un délai de cinq mois. On peut donc dire que $34 \%$ des réponses ont été faites dans un délai de deux mois et $55 \%$ dans un délai de trois mois. Les réponses obtenues après plus de trois mois représentent $45 \%$ du total des réponses.

2. Drion, Françoise, «Les questions parlementaire écrites», Revue de l'Institut belge de science politique, XVII, 1975, 208.

3. Nguyen Huu, Patrick, "L'évolution des questions parlementaires depuis 1958", Revue française de science politique, février 1981, 173-175.

4. Règlement de l'Assemblée nationale du Québec (mai 1981), art. 169: «Aucune question écrite ne peut être posée à moins qu'il n'en ait été donné avis suivant les règles relatives aux avis de motion. » 
TABLEAU 2

Répartition des questions écrites selon les partis et le délai entre l'avis et la réponse (1979-1980)

\begin{tabular}{lccccc}
\hline & PLQ & UN & PQ & AUTRE & TOTAL \\
$0-1$ mois & $2(7 \%)$ & $3(11 \%)$ & $1(20 \%)$ & & $6(9 \%)$ \\
$1-2$ mois & $9(32 \%)$ & $7(26 \%)$ & & & $16(25 \%)$ \\
$2-3$ mois & $5(18 \%)$ & $8(30 \%)$ & & & $13(21 \%)$ \\
$3-5$ mois & $4(15 \%)$ & $5(19 \%)$ & $1(20 \%)$ & $3(100 \%)$ & $13(21 \%)$ \\
5 mois et plus & $8(29 \%)$ & $4(15 \%)$ & $3(60 \%)$ & & $15(24 \%)$ \\
\cline { 2 - 6 } & $28(100 \%)$ & $27(100 \%)$ & $5(100 \%)$ & $3(100 \%)$ & $63(100 \%)$
\end{tabular}

En analysant le délai de réponse, nous n'avons pas considéré les questions écrites qui sont demeurées sans réponse. Ces questions étaient au nombre de dix en 1979-1980, ce qui représentait un taux de réponse de $86 \%$. Toutefois, deux de ces questions ont été inscrites très tard, le 5 juin 1980, soit en pleine période de fin de session. Une autre portait sur une hypothèse qui ne s'est pas réalisée, d'où l'absence de réponse ${ }^{5}$. En enlevant ces trois questions, on arrive à un taux de réponse de $90 \%$.

En France, le taux de réponse aux questions écrites était à l'Assemblée nationale de $90 \%$, en 1969 , et de $95 \%$, en $1979^{6}$.

En Belgique, en ce qui concerne la Chambre, pour la session 1970-1971, sur un total de 2,381 questions, 48 d'entre elles n'ont pas reçu de réponse, soit $2 \%$ des questions. Pour la session 1971-1972, sur un total de 1,528 questions, 59 sont demeurées sans réponse, soit $4 \%$ des questions. «Ne perdons pas de vue que ces relevés ne mentionnent pas la multitude de questions qui ont reçu une réponse provisoire et à laquelle il n'est souvent pas donné suite ${ }^{7}$. »

5. Coûts aux municipalités suite au changement des panneaux «arrêt stop».

6. Nguyen Huu, op. cit., 175.

7. Drion, Françoise, op. cit., 219. 


\section{Publicité des questions écrites}

Les avis des questions écrites sont publiés dans le Feuilleton de l'Assemblée nationale du Québec. Tant qu'une question écrite n'a pas été l'objet d'une réponse, elle demeure au Feuilleton, précédée de la date de l'avis. Les questions écrites non répondues constituent, à chaque mercredi, le premier sujet des affaires du jour inscrites au Feuilleton ${ }^{8}$. Cette procédure peut représenter en quelque sorte un moyen d'incitation auprès des ministres pour qu'ils fournissent les informations demandées.

Quant aux réponses, elles apparaissent dans les Procèsverbaux de l'Assemblée nationale du Québec accompagnées des questions correspondantes. En général, au cours de la période étudiée, la réponse à une question écrite fut donnée le mercredi, sauf à 15 reprises où ce fut un jeudi. Lorsque la réponse est trop longue pour être publiée dans les Procies-verbaux, le ministre peut décider de la transformer en dépôt de document. Ce document est classé comme document de la session et il n'est transmis aux députés, aux journalistes et au public en général que sur demande. Il n'y a que le député qui a posé la question qui reçoit directement une copie du document. En 1979-1980, 41 réponses ont été transformées en dépôt de document, ce qui représente $65 \%$ de toutes les réponses.

La diffusion des Procès-verbaux ne nous permet pas de juger de l'intérêt de cette procédure parlementaire. En effet, les réponses aux questions écrites ne constituent qu'un élément du grand ensemble que sont les Pročs-verbaux. Au contraire, en France, il y a une édition spéciale du Journal officiel qui est consacrée uniquement aux questions écrites. Également, en Belgique, il existe un recueil hebdomadaire des questions écrites, soit le Bulletin des Questions et réponses.

8. Voir l'art. 35-2 (a) du Règlement de l'Assemblée nationale du Québec (mai 1981). 


\section{Conclusion}

Au Québec, les questions écrites sont accessibles à tous les parlementaires, qu'ils appartiennent à la majorité ou à l'opposition. Cette dernière questionne en vue d'obtenir des informations qu'elle ne peut se procurer autrement et ainsi contrôler éventuellement certains actes de l'administration, tandis que les députés ministériels utilisent les questions soit pour obtenir des informations relatives à leur circonscription électorale, soit pour attaquer l'administration précédente. Ici, contrairement à la France, les questions écrites sont soumises au rythme des sessions et ainsi elles ne permettent pas au contrôle parlementaire de s'exercer de façon continue, en session et hors session. Par exemple, les députés ne peuvent pas employer cette procédure lors des vacances estivales et des fêtes, une période d'environ cinq mois, durant laquelle de nombreux actes administratifs sont posés.

C'est sûrement une des raisons qui explique le nombre peu élevé des questions écrites à l'Assemblée nationale du Québec comparativement à d'autres parlements. En 1979-1980, les 110 députés de l'Assemblée ont posé un total de 73 questions écrites, ce qui se traduit par une moyenne de 0,6 question par député $^{9}$. Du côté de la Belgique, en 1972-1973, les 212 députés ont demandé 2365 questions écrites, ce qui représente une moyenne de 11 questions par député. En France, les 491 députés de l'Assemblée nationale ont soulevé 15920 questions écrites, en 1980, ce qui donne une moyenne de 32 questions par député. D'ailleurs le nombre élevé des questions écrites en France est le motif principal de la récente décision du bureau de l'Assemblée

9. De l'avis de Mlle Constance Pinault, au secrétariat général de l'Assemblée, les questions écrites ont été plus nombreuses en 1977 et 1978 (de 2, 5 à 3 questions par député). Elle considère ces deux années comme exceptionnelles à ce point de vue, cela étant dû au fait que l'opposition officielle avait assigné un préposé à la rédaction des motions et des questions. 
nationale de leur consacrer une édition spéciale du Journal officiel ${ }^{\text {10 }}$.

Les délais de réponse trop longs représentent un obstacle sérieux au succès de la procédure des questions écrites. Un délai de deux mois, au maximum trois, devrait être suffisant aux ministres. Le contenu des réponses, même s'il n'a pas fait l'objet d'une analyse de notre part, peut de toute évidence constituer également une entrave au bon fonctionnement des questions écrites.

Les questions écrites sont loin de connaître la même publicité que les questions orales. On sait que ces dernières sont télévisées. D'autre part une analyse de la presse quotidienne nous démontre que celle-ci ne fait pas souvent écho dans ses colonnes aux questions écrites ${ }^{11}$. À première vue, deux raisons peuvent expliquer cette absence: d'abord lorsque la réponse est donnée, sauf exception, le problème n'est plus d'actualité ; également les questions concernent souvent un individu en particulier et ainsi l'intérêt général est moins évident. De toute façon, des parlementaires désireux de se donner une plus large publicité ne devraient-ils pas signaler aux journalistes qu'ils se sont intéressés à tel ou tel problème, surtout lorsque les réponses sont transformées en dépôt de document, en conséquence moins accessibles? Vue sous cet angle, la question écrite apparaîtrait comme un moyen d'information du grand public. Mais encore là, si la réponse ne démontre pas une faiblesse de l'Administration, le député de l'opposition n'aura rien à gagner à la faire connaître.

10. Drion, Françoise, op. cit., 204, 211, 219. Nguyen Huu, op. cit., 172173.

11. La méthodologie de cette analyse a consisté simplement à faire le choix d'un échantillonnage d'une vingtaine de questions écrites, une moitié faisant l'objet d'une réponse directe, l'autre moitié faisant l'objet d'une réponse transformée en dépôt de document. L'analyse de presse s'est faite le lendemain de la parution de la réponse dans les Procès-verbaux de l'Assemblée. Les journaux choisis ont été Le Soleil, Le Journal de Québec, La Presse et Le Devoir. 
Quant au sujet des questions écrites, nous devons souligner le même paradoxe que nous avions déjà constaté dans une autre étude qui portait sur les questions avec débat ${ }^{12}$ : les parlementaires négligent les sujets reliés au domaine du social et de l'éducation, alors que ces derniers engouffrent la plus grande part du gâteau budgétaire gouvernemental. Notons également que la mission économique fut plus scrutée au moyen des questions avec débat ( $40 \%$ du total des questions) que lors des questions écrites (18\%). Au contraire, les parlementaires ont accordé plus de soin à la mission gouvernementale et administrative au moyen des questions écrites $(67 \%)$ que lors des questions avec débat $(40 \%)^{13}$. Qu'est-ce qui a amené les députés à concentrer à ce point leurs questions écrites sur cette dernière mission? Le référendum, même s'il a eu lieu durant la session étudiée, n'a pas fait l'objet de plusieurs questions écrites. Ce sont plutôt des questions touchant à un individu en particulier qui ont constitué plus de la moitié de ces questions.

12. Champagne, Maurice. La Question avec débat à l'Assemblée nationale du Québec. Québec, Bibliothèque de l'Assemblée nationale, Division de la recherche, juin 1981, 11. (inédit)

13. L'étude sur les questions écrites s'étend sur une période plus courte (mars 1979 à juin 1980) que celle sur les questions avec débat (1977-1980). Cependant, même en réduisant cette dernière à la période couverte par les questions écrites, la tendance demeure. 